\title{
Necrotizing Fasciitis of Face in Odontogenic Infection: A Rare Clinical Entity
}

\author{
GK Vivek, Ranjith Singh, GC Veena, Prappanna Arya
}

\begin{abstract}
Necrotizing fasciitis is a progressive, life-threatening, bacterial infection of the skin, the subcutaneous tissue and the underlying fascia, in most cases caused by $\beta$-hemolytic group $A$ Streptococcus. Only early diagnosis and aggressive therapy including broad spectrum antibiotics and surgical intervention can avoid systemic toxicity with a high mortality rate. This disease is commonly known to occur in the lower extremities and trunk, and only rarely in the head and neck region, the face being rarest finding. When located in the face necrotizing fasciitis is associated with severe cosmetic and functional complication due to the invasive nature, infection and often due to the necessary surgical treatment. In the following article, we present the successful diagnosis and management of an isolated facial necrotizing fasciitis as a consequence of odontogenic infection.
\end{abstract}

Keywords: Necrotizing fasciitis, Facial region, Immunocompromised, Odontogenic infection.

How to cite this article: Vivek GK, Singh R, Veena GC, Arya $P$. Necrotizing F asciitis of $F$ ace in O dontogenic Infection: A Rare Clinical Entity. World J Dent 2012;3(3):261-264.

\section{Source of support $\mathrm{Nil}$}

Conflict of interest: None declared

\section{INTRODUCTION}

Necrotizing fasciitis was first described in 1848. In 1920, $M$ eleney identified 20 patients in China in whom hemolytic Streptococcus was the sole organism. Wilson coined the term necrotizing fasciitis in 1952 and found no specific pathologic bacteria related to the disease. ${ }^{1} \mathrm{~N}$ ecrotizing fasciitis is characterized by fulminating, devastating and rapidly progressing condition, with a high morbidity and mortality rate. The disease is now known to be caused by a polymicrobial or mixed aerobic-anaerobic infection. ${ }^{2}$ Patients with immunocompromised status like diabetes mellitus, cancer, alcoholism, vascular insufficiencies, organ transplants, HIV or malnutrition are prone to this type of infection. ${ }^{3,4}$ O rganisms spread from the subcutaneous tissue along the superficial and deep fascial planes, presumably facilitated by bacterial enzymes and toxins. This deep infection causes vascular compression, ischemia and tissue necrosis. Superficial nerves are damaged, producing the characteristic localized anesthesia. ${ }^{5,6}$ Septicemia ensues with systemic toxicity. Necrotizing fasciitis seen commonly seen in the extremities, trunk and perineum. These infections can be difficult to recognize in their early stages and require aggressive treatment to combat the associated high morbidity and mortality.
Facial and cervical necrotizing fasciitis though a rare entity, usually results from progressive, suppurative dental infections, other etiologies include peritonsillar abscess, salivary gland infections, otologic and dermatologic sources. The infections may easily spread via natural fascial planes into deep cervical spaces or vascular compartments. M any complications of cervical necrotizing fasciitis have been described, including airway obstruction, jugular venous thrombosis, rupture of the great vessels, aspiration pneumonia, mediastinitis, empyema, and lung abscess, if it occurs in the neck due to the close proximity and rapid spread through facial planes.

\section{CASE REPORT}

A 52-year-old lady by named lakshmamma reported to us with a history of chronic buccal space infection. The patient gave history of swelling since 6 months and had been prescribed repeated doses of antibiotics which included amoxicillin, amoxicillin with clavulanic acid and ciprofloxacillin antibiotics, which she continued to take multiple times without any surgical treatment. The patient on reporting was al most going for septic shock (Figs 1 and 2),

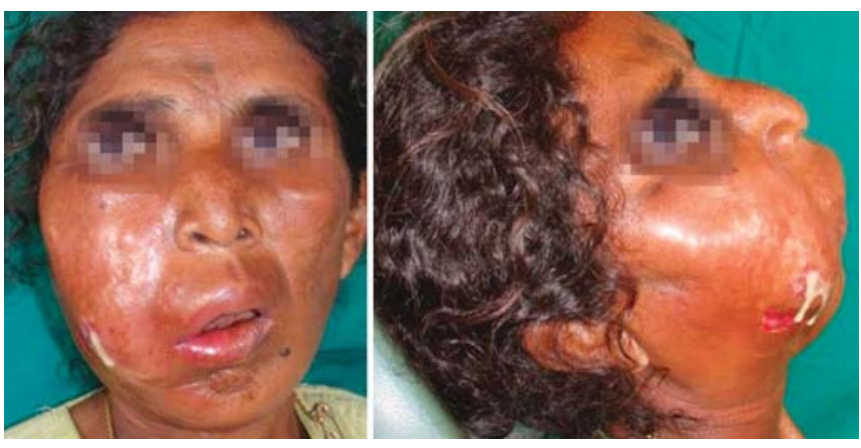

Fig. 1: Patient on presentation

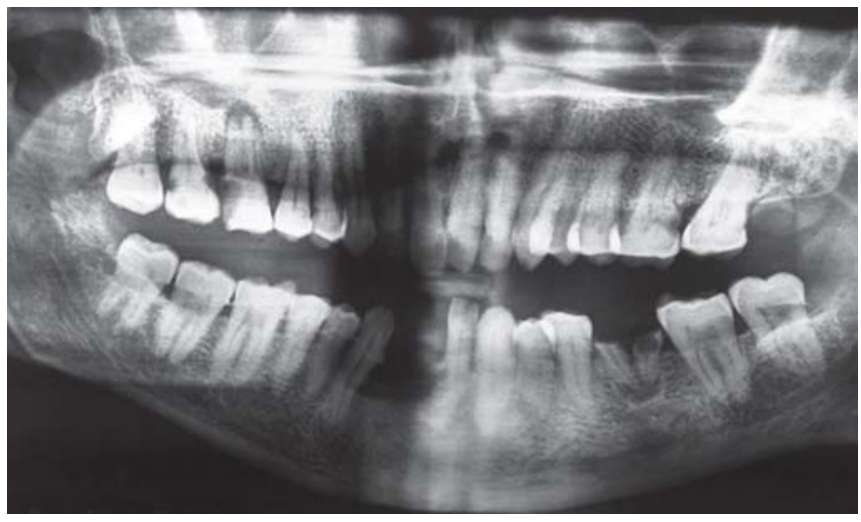

Fig. 2: Preoperative orthopantomogram (OPG) showing periapical infection of 16 tooth 


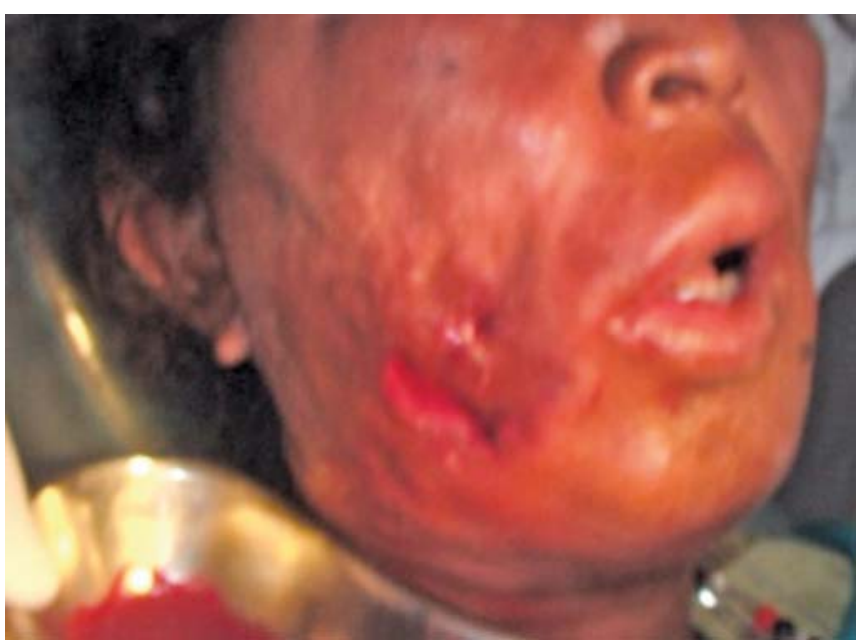

Fig. 3: Intraoperative picture of I\&D

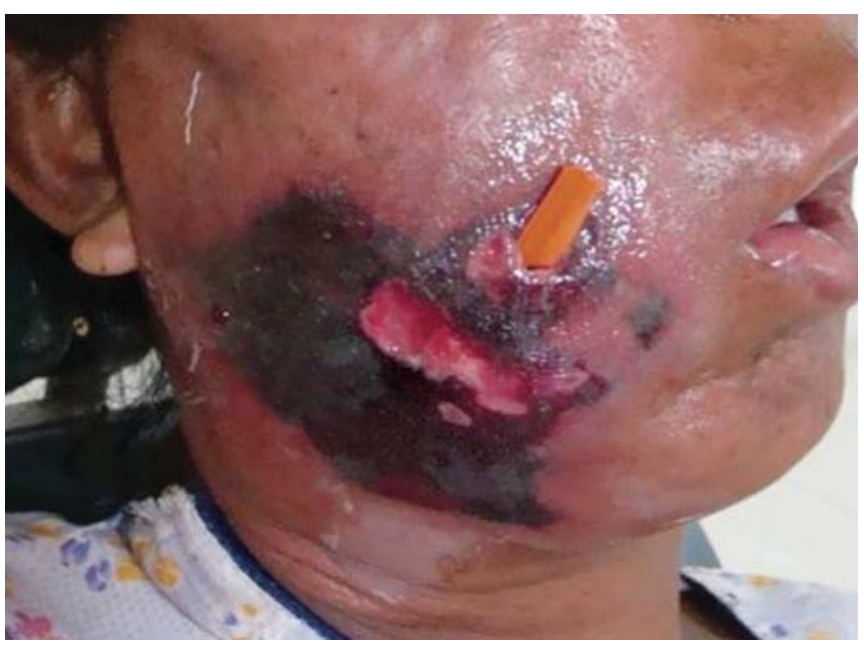

Fig. 4: Postoperative picture showing developing necrotizing fasciitis

patient was immediately put on systemic antibiotics cefotaxime $1 \mathrm{gm}$, and metronidazole $500 \mathrm{mg}$ and immediate incision and drainage of swelling done through the extraoral draining sinus over the right cheek (Fig. 3). The offending maxillary right first molar tooth was extracted under local anesthesia, the patient progressed to necrotizing fasciitis over right cheek (Figs 4 and 5) dueto chronic infection for a period of about 4 months, the other cause for such a rare finding can be attributed to the fact the patient hailed from poor socioeconomic status and was grossly malnourished. The patient was treated by surgical drainage and empirical antibiotic therapy following culture sensitivity report which indicated mixed infection containing both gram-positive cocci and gram-negative rods. She was treated by daily by topical wound debridement followed with drainage and correcting the malnutrition with multivitamins along with protein supplements following which patient recoverd uneventfully (Fig. 6).

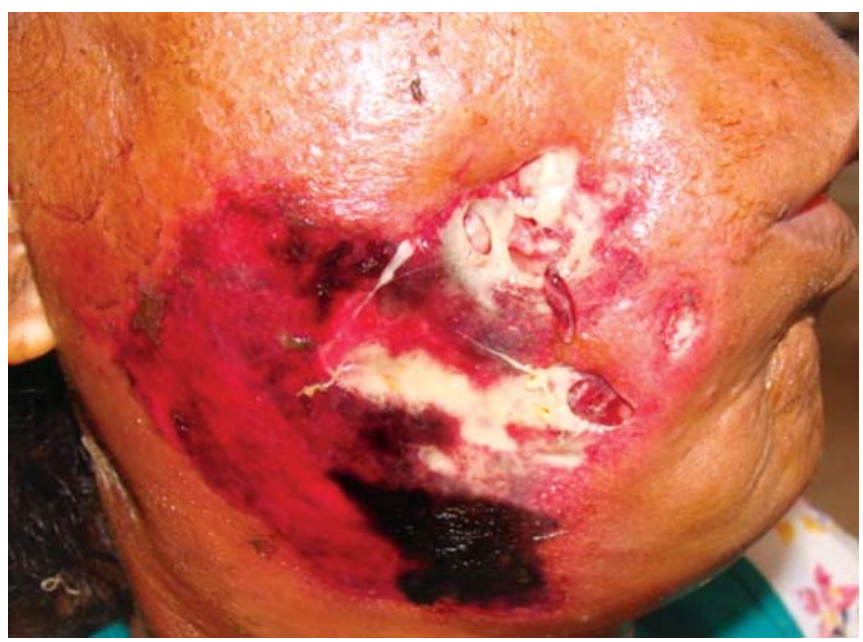

Fig. 5: Full blown necrotizing fasciitis of right cheek

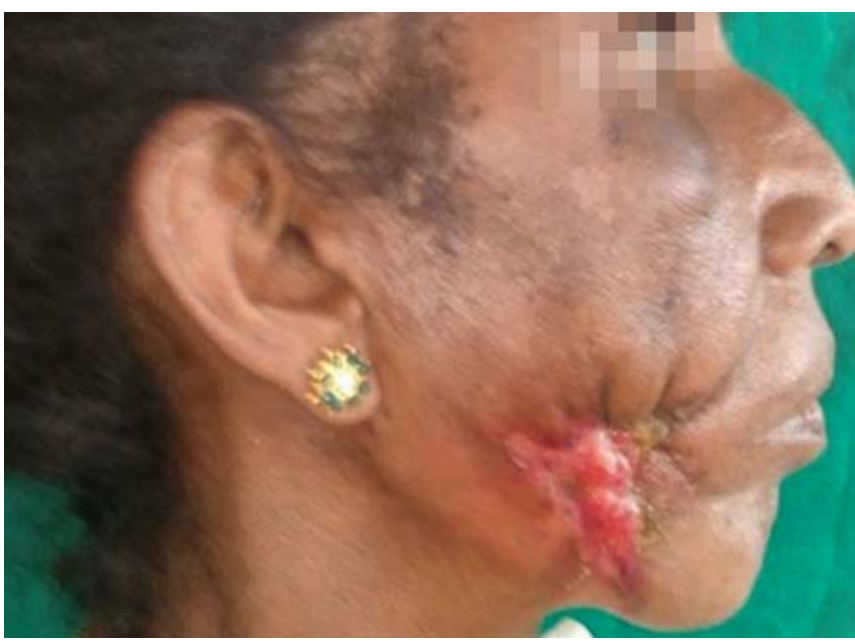

Fig. 6: 10th day postoperative follow-up picture of patient

\section{DISCUSSION}

Necrotizing fasciitis has been described under various synonyms, including hospital gangrene, hemolytic or acute streptococcal gangrene, gangrenous or necrotizing erysipelas, suppurative fasciitis, M eleney's gangrene and Fournier's gangrene. ${ }^{3-6,9-12}$ The term necrotizing fasciitis (NF) was first coined by Wilson in $1952 .{ }^{3,6} \mathrm{NF}$ is usually known to occur in the perineum, lower limbs or abdominal wall following surgery or trauma, particularly in individuals with underlying systemic diseases, such as diabetes mellitus, arteriosclerosis, chronic renal failure or malnutrition ${ }^{1,3,4} \mathrm{NF}$ may affect patients of all ages, without sex or race predilection. ${ }^{3,6} \mathrm{NF}$ is rarely seen in the head and neck region, ${ }^{1,6}$ however, its occurrence is probably more than the reported cases would suggest NF involvement of the scal p and face is less common when compared to the cervical region. In the majority of NF cases involving the neck, the disease follows dental or oropharyngeal infection, ${ }^{1-3,6}$ 
Necrotizing Fasciitis of Face in O dontogenic Infection: A R are Clinical Entity

whereas in the cases of scalp or face involvement, there is mostly a previous trauma that might be minor external evidence of the infection. $3,5,7,11$

Overall incidence of necrotizing fasciitis is approx 1 in 453,333 or $0.00 \%$ or 600 people/year in USA, ${ }^{12-14}$ the clinical picture is dependent on the stage of the disease and its primary origin. Several authors have reviewed the presentation and the management of the disease. ${ }^{2,5,6,7,14} \mathrm{~T}$ he clinical signs and symptoms are nonspecific. The skin is red-hot, smooth, tense and tender without sharp demarcation between the involved and normal skin. Crepitation, may be elicited. A s the disease progresses, the skin becomes dusky and small blisters appear, our patient reported to us when she had developed these symptoms, ultimately, frank gangrene takes place. The skin necrosis is secondary to thrombosis of the nutrient vessels passing through necrotic fascia. ${ }^{1-4,11}$ The patient is acutely ill, with low to medium grade fever associated with tachycardia. All fascial planes of the head and neck can be involved in NF and may not be localized. A vascularity of the fascial planes is probably the cause of their involvement and initially sparing the muscles and skin. ${ }^{3,4}$ B acterial necrotoxins might play a role in tissue damage. The hallmarks of NF are widespread fascial necrosis with extensive undermining and serosanguinous, often foul-smelling exudate. ${ }^{1-6,7}$ Passing a blunt instrument easily along the fascial plane is a specific clinical sign, a spectrum of microbes have been reported to be the primary pathogens. ${ }^{1-6,9}$ The microorganisms isolated in our cases are consistent with the view that NF is a synergetic bacterial infection usually produced by a combination of grampositive cocci and gram-negative rods. ${ }^{3,6}$

Prompt and successful management of NF is universally agreed to be aggressive surgical intervention, intravenous wide-spectrum antibiotics and supportive therapy. It is of the utmost importance to explore and drain all affected areas. Wide extensive fasciotomies with exposure of all involved fascia and excision of necrotic tissues should be carried out. Early surgical intervention would minimize loss of the covering skin and avoid cosmetic disfiguring. ${ }^{4-6,8}$ The heal thy skin should not be excised initially, even in extensive debridement of the underlying fascia. Skin necrosis occurring late can be dealt with later, adopt a wait and watch policy when dealing with such extensive skin involvement of face region. Broad-spectrum antibiotics covering both aerobic and anaerobic organisms should be initiated and changed according to the sensitivity patterns. We would like to stress the point that antibiotic therapy alone is considered insufficient for the management of NF without surgical intervention. ${ }^{1-7}$ The nutritional support, whether enteral or parenteral, is also of great importance. The local and systemic complications of N F are numerous and include extension to the cervical viscera and mediastinum, vessel erosion and functional and cosmetic disfiguring. ${ }^{1,3}$ Death from necrotizing fasciitis is usually due to overwhelming sepsis, respiratory failure, renal failure or multiorgan failure. ${ }^{3,6,7}$ The mortality rate ranges between 8 and $73 \%$.,3,6 The principal factors contributing to the high mortality are delayed diagnosis and treatment, extent of the disease, old age and associated systemic illnesses. ${ }^{1-5}$ N ecrotizing fasciitis involving the scalp and upper face has better prognosis than that involving the neck but it is more grue some and has a psychological impact on patient. ${ }^{3}$ The presented case emphasize the need for early diagnosis, proper aggressive surgical intervention and intravenous antibiotic treatment of this serious life-threatening infection. The need to evaluate the systemic condition of the patient plays a vital role as the odontogenic infection alone cannot cause such a severe infection in the absence of a debilitating condition. The development of necrotizing fasciitis must be recognized as early as possible to prevent unnecessary morbidity and mortality and no infection odontogenic or nonodontogenic should be taken for granted as it has far reaching effects.

\section{ACKNOWLEDGMENT}

Dr Shyla N, Head, Department of Oral and M axillofacial Surgery, KLEDC, B engaluru.

\section{REFERENCES}

1. Tung Yiu W, Jehn Shyun H, Ching Hung C, Hung An C. Cervical necrotizing fasciitis of odontogenic origin, a report of 11 cases. J Oral M axillofac Surg 2000 Dec;58:1347-52.

2. Obiechina AE, A rotiba JT, Fasola A O. N ecrotizing fasciitis of odontogenic origin in Ibadan, N igeria. BrJ Oral M axillofac Surg 2001A pr;39:122-26.

3. Whitesides L, Cotto C umba C, M yers RA. Cervical necrotizing fasciitis of odontogenic origin, a case report and review of 12 cases. J Oral M axillofac Surg 2000 Feb;58:144-51.

4. Raboso E, Llavero MT, Rosell A, M artinez-Vidal A. Craniofacial necrotizing fasciitis secondary to sinusitis. J L aryngol Otol $1998 \mathrm{~A} \mathrm{pr;112:371-72.}$

5. Fliss DM, Tovi F, Zirkin HJ . N ecrotizing soft-tissue infections of dental origin. J Oral M axillofac Surg 1990 0 ct;48:1104-08.

6. B axter $C R$. Surgical management of soft tissue infections. Surg Clin N orth A m 1972;52:1483-99.

7. Rapoport Y, Himelfarb MZ, Zikk D, Bloom J. Cervical necrotizing fasciitis of odontogenic origin. Oral Surg Oral M ed Oral Pathol 1991;72:15-18.

8. Y amaoka M, Furusawa K, Kiga, M, Iguchi K, Hirose I. Necrotizing buccal and cervical fasciitis. J Cranio Maxillofac Surg 1990;18:223-24.

9. Drake-L ee A B , B roughton SJ, Rampling A, L ancer J M , M offat DA.N ecrotizing fasciitis. J Laryngol Otol 1983;97:193-96. 
10. Maisel RH, Karlen R. Cervical necrotizing fasciitis. Laryngoscope 1994;104:795-98.

11. Sepúlveda A, Sastre N. Necrotizing fasciitis of the face and neck. Plast Reconstr Surg 1998 Sep;102(3):814-17.

12. www .rightdiagnosis.com/n/necrotizing fasciitis/ prevalence.htm.

13. www.medicinenet.com/necrotizing_fasciitis/page.7.htm.

14. Joseph Brunworth M d, Terry $Y$, Shibuya M d. Craniocervical necrotizing fasciitis resulting from dentoal veolar infection. Oral Maxillofacial Surg Clin N A m 2011;23:425-32.

\section{ABOUT THE AUTHORS}

\section{GK Vivek (Corresponding Author)}

Senior L ecturer, D epartment of O ral and M axillofacial Surgery, KLE Institute's of Dental Sciences College and Hospital, No. 513 12th M ain R oad, Sector A, Y elahanka N ew town, B engaluru-560106 Karnataka, India, e-mail: vivekbhatomfs@gmail.com

\section{Ranjith Singh}

Reader, Department of Oral and M axill ofacial Surgery, KLE Institute's of D ental Sciences College and H ospital, B engal uru, K arnataka, India

\section{GC Veena}

Senior Lecturer, Department of Oral and M axillofacial Surgery, KLE Institute's of Dental Sciences College and Hospital, B engaluru Karnataka, India

\section{Prappanna Arya}

Senior L ecturer, Department of Oral and M axillofacial Surgery, KLE Institute's of Dental Sciences College and Hospital, B engaluru Karnataka, India 